\title{
Early Cyclosporine A Withdrawal in Kidney- Transplant Recipients Receiving Sirolimus Prevents Progression of Chronic Pathologic Allograft Lesions
}

\author{
Juan C. Ruiz, ${ }^{1,11}$ Josep M. Campistol, ${ }^{2}$ Josep M. Grinyó, ${ }^{3}$ Alfredo Mota, ${ }^{4}$ Dolores Prats, ${ }^{5}$ Jose A. Gutiérrez, ${ }^{6}$ \\ Antonio C. Henriques, ${ }^{7}$ Jose R. Pinto, ${ }^{8}$ Javier García, ${ }^{9}$ Jose M. Morales, ${ }^{10}$ Jose M. Gómez, ${ }^{1}$ and \\ Manuel Arias ${ }^{1}$
}

\begin{abstract}
Background. Nephrotoxicity of calcineurin inhibitors (CNIs) is partially responsible for the development of chronic allograft nephropathy (CAN). Sirolimus has demonstrated its potential to substitute for CNIs because it lacks significant nephrotoxicity and shows a short-term immunosuppressive capacity comparable with that of cyclosporine. This results in the maintenance of better renal function when cyclosporine is eliminated, but it has not been demonstrated whether this benefit is associated with an improvement in the pathologic substrate and a reduction in CAN.

Methods. We analyzed pretransplant and 1-year renal-allograft biopsies from 64 patients enrolled in a multicenter trial. Patients received cyclosporine and sirolimus during the first 3 months after transplant and were then randomly assigned to continue with cyclosporine or have it withdrawn. Histologic chronic allograft lesions were compared between groups.

Results. The percentage of patients in whom chronic pathologic lesions progressed was lower in the group of cyclosporine elimination. Significant differences were observed in chronic interstitial and tubular lesions $(70 \%$ vs. $40.9 \%$ $[P<0.05]$ and $70 \%$ vs. $47.8 \%[P<0.05]$, respectively), whereas no differences were observed in acute lesions (subclinical rejection). Prevalence of CAN at 1 year was lower in this group, as was the severity and incidence of new cases $(P<0.05)$. Conclusions. Early cyclosporine withdrawal associated with sirolimus administration is followed by an improvement in renal function, a reduction in the progression of chronic pathologic allograft lesions, and a lower incidence of new cases and severity of CAN during the first year after transplantation. This benefit may result in better long-term graft outcome.
\end{abstract}

Keywords: Sirolimus, Cyclosporine A withdrawal, Acute rejection, Renal function, Chronic allograft nephropathy.

(Transplantation 2004;78: 1312-1318)

G iven the considerably high short-term success rate obtained with conventional immunosuppressive drugs (calcineurin inhibitors [CNIs]), the objectives in the development of new drugs during the last few years have focused on long-term results, specifically on the prevention of chronic graft damage (1). Chronic allograft nephropathy (CAN) is the main cause of graft loss after the first year of transplantation, with a prevalence of $60 \%$ to $70 \%$ in protocol biopsies at 2 years after transplantation in patients receiving CNIs, either cyclosporine A (CsA) or tacrolimus, without significant differences between the drugs (2). The role of these drugs in the development of CAN and subsequent long-term graft sur-

This work was supported by a grant from Wyeth Farma S.A., Madrid, Spain.

${ }^{1}$ Hospital Universitario Marqués de Valdecilla, Santander, Spain.

${ }^{2}$ Hospital Clinic i Provincial, Barcelona, Spain.

${ }^{3}$ Hospital Bellvitge, Barcelona, Spain.

${ }^{4}$ Hospital Da Universidade De Coimbra, Coimbra, Portugal.

${ }^{5}$ Hospital Clínico, Madrid, Spain.

${ }^{6}$ Hospital Miguel Servet, Zaragoza, Spain.

${ }^{7}$ Hospital Geral de Santo Antonio, Porto, Portugal.

${ }^{8}$ Hospital Curry Cabral, Lisboa, Portugal.

${ }^{9}$ Hospital La Fe, Valencia, Spain.

${ }^{10}$ Hospital 12 de Octubre, Madrid, Spain.

11 Address correspondence to: Juan Carlos Ruiz San Millán, Servicio de Nefrología, Hospital Universitario Marqués de Valdecilla 39008 Santander, Spain.

Received 5 November 2003. Accepted 21 May 2004.

Copyright $\odot 2004$ by Lippincott Williams \& Wilkins

ISSN 0041-1337/04/7809-1312

DOI: 10.1097/01.TP.0000137322.65953.0A vival seems to be of major importance because of their nephrotoxic effect (3).

The use of new drugs with similar immunosuppressive capacity but without the nephrotoxic effect may yield a considerable advance in increasing long-term graft survival. Sirolimus (SRL, rapamycin, Rapamune) has shown similar efficacy to CsA when used alone with respect to graft rejection rates and short-term graft survival in combination with either steroids and azathioprine (AZA) (4) or steroids and mycophenolate mofetil (5). Similar results have been observed when adding induction therapy with basiliximab (6). In addition, significantly better renal function has been consistently observed in patients treated with SRL but without CNIs $(4-7)$. The association of CsA and SRL has a synergistic and potent immunosuppressive effect with very low rejection rates, but SRL appears to potentiate CsA nephrotoxicity because of a pharmacokinetic interaction. Clinical trials analyzing this combination showed worse renal function in the groups in which the two drugs were combined compared with the control group of CsA plus AZA or placebo $(8,9)$.

With the objective to obtain a low rejection rate and the minimization of the long-term deleterious effects of CsA nephrotoxicity, a multicenter, randomized, phase 3 trial at 5 years is currently ongoing in 57 centers in Europe, Canada, and Australia, with a total of 525 patients enrolled (The Rapamune Maintenance Regimen [RMR] Study). This trial compares a control arm (group A) using CsA, fixed doses of SRL, and steroids with a study arm (group B) that includes CsA, concentration-controlled SRL, and steroids with elimi- 
nation of CsA at 3 months after transplantation. The initial results from this trial have been previously published and demonstrated significantly better graft function (lower serum creatinine and higher calculated glomerular filtration rate) at 1 year in group B (10). However, it has not yet been clearly demonstrated whether this improvement in renal function is a consequence of a hemodynamic improvement caused by the prevention of CsA functional vascular effects or whether the initial expression of a reduction in chronic tubulointerstitial and vascular damage may be responsible for a reduction in the occurrence or severity of CAN in the long term and a subsequent increase in allograft survival.

We previously attempted to address this question in a substudy of the RMR trial. We evaluated 140 renal biopsies in patients with a functioning allograft at 1 year, corresponding with the baseline (pretransplant) and 1-year biopsies of the 70 patients recruited in the 10 centers participating in Spain and Portugal (11). In that substudy, we performed a retrospective analysis of the information supplied by the 10 local pathologists (according to the Banff'95 classification) and observed a lower rate of progression of chronic allograft pathologic lesions when CsA was eliminated. This type of analysis, however, had implicit methodological difficulties to overcome. We could not verify that local pathologists read the biopsies in a blinded manner with respect to treatment arm and time of biopsy. Some lesions could have been interpreted differently, whether they appeared in the pretransplant biopsy or 1-year biopsy. Furthermore, the participation of 10 different pathologists in the interpretation of biopsies may have been responsible for a significant interobserver variation in the interpretation of the existence and severity of elemental chronic lesions. These shortcomings have been extensively evaluated by Furness and Taub (12) in the Convergence Of European Renal Transplant Pathology Assessment Procedures (CERTPAP) Project, who recommend caution when interpreting biopsy results between institutions.

Therefore, we performed a prospective and more profound reanalysis of the renal biopsies of this subgroup of patients, amplifying the histologic analysis, which was now performed by a central blinded pathologist. This article presents the results of this reanalysis comparing the severity of chronic pathologic allograft lesions in the two treatment groups.

\section{MATERIALS AND METHODS}

\section{Rapamune Maintenance Regimen Trial}

The RMR Trial is a multicenter, randomized, openlabel study that was initiated in May 1998 at 57 centers in Europe, Canada, and Australia. All centers obtained approval from their local ethics committees, and each patient enrolled signed an informed consent form before transplantation. Immunosuppression included CsA, SRL at fixed doses $(2 \mathrm{mg} /$ day), and steroids for all patients during the first 3 months after transplantation. Patients were then randomly assigned to one of two treatment arms: patients in group A continued receiving CsA, SRL $2 \mathrm{mg}$, and steroids, whereas patients in group B had CsA progressively decreased and then eliminated in approximately 4 to 6 weeks and SRL increased to maintain SRL trough levels in the range of 16 to $24 \mathrm{ng} / \mathrm{mL}$ by highperformance liquid chromatography-ultraviolet (HPLCUV) or HPLC-mass spectrometry methods (20 to $30 \mathrm{ng} / \mathrm{mL}$ by enzyme-linked immunoadsorbent assay method). Patients with Banff grade III acute rejection or vascular rejection in the 4 weeks before randomization, dialysis dependency, serum creatinine greater than $400 \mu \mathrm{mol} / \mathrm{L}$, or inadequate renal function to support CsA elimination were excluded from randomization. Patients underwent a pretransplant biopsy, a second biopsy at 1 year after transplantation, and a third biopsy at 3 years after transplantation. This study remains ongoing, with follow-up of patients through the fifth year after transplantation. Details of the study design and clinical results at 1 year were published by Johnson et al. (10), and additional information with follow-up at 2 years has been published recently by Oberbauer et al. (13).

\section{Patients}

All 96 patients enrolled in the RMR Trial centers in Spain and Portugal were considered for inclusion in the present substudy. Patients who lost their grafts within the first year after transplantation and those without an evaluable biopsy at the time of transplantation or at 1 year thereafter were excluded from the study. Seventy patients had a functioning graft at 1 year, and kidney biopsies were performed at the time of transplantation and at 1 year after transplantation. Sixtyfour $(91.4 \%)$ of those patients were evaluable (in 6 cases, biopsy material could not be recovered for evaluation). All patients included in this substudy received kidney transplants from cadaveric donors. Table 1 shows the demographic characteristics of patients included in the analysis. Both groups were comparable for all data considered.

\section{Evaluation of Renal Function}

Renal function was analyzed at 1 year after transplantation using serum creatinine and calculated creatinine clearance (Nankivell method). Both parameters were compared between treatment arms. Given the similar findings for these two determinations, we will only present the serum creatinine results.

\section{Pathologic Evaluation}

All patients who were evaluated had two kidney-graft biopsies, the first at the time of transplantation and the second at 1 year after transplantation. The 128 graft biopsies were processed using conventional techniques and staining with hematoxylineosin, periodic-acid Schiff, and Masson's trichrome. All slides were evaluated by a central pathologist (author JMG) who was blinded to the time of collection of the biopsy and the treatment arm. Each biopsy was evaluated according to the Banff 1997 working classification (14) and the Chronic Allograft Damage Index (CADI) classification (15). A Banff chronicity score (Banff-CS) was calculated as the sum of the four chronic basic lesions (chronic glomerular damage $[c g]+$ interstitial fibrosis $[c i]+$ tubular fibrosis $[c t]+$ vascular intimal thickening $[c v])$, and a CADI score was calculated as the sum of the six parameters evaluated in this classification (inflammation $[I]+$ interstitial fibrosis $[F]+$ mesangial matrix increase $[M]+$ glomerular sclerosis $[G]+$ tubular atrophy $[T]+$ intimal proliferation $[V])$. Acute lesions were also evaluated using the Banff criteria and were compared between treatment arms with the objective of detecting differences in inflammatory activity, the so-called subclinical rejection, responsible for theoretical future chronic damage in the long term. 
TABLE 1. Demographic parameters and characteristics of patients and biopsy specimens in both treatment arms

\begin{tabular}{|c|c|c|c|c|}
\hline Characteristic & Group A & Group B & Total & $P$ Value \\
\hline Sex, n (\%) & & & & $0.385^{a}$ \\
\hline Male & $24(80)$ & $24(71)$ & $48(75)$ & \\
\hline \multicolumn{5}{|l|}{ Ethnic origin, $\mathrm{n}(\%)$} \\
\hline White & $30(100)$ & $34(100)$ & $64(100)$ & \\
\hline \multicolumn{5}{|l|}{ Age (years) } \\
\hline Mean (range) & $44.4(22-66)$ & $47.1(18-66)$ & $45.8(18-66)$ & $0.352^{b}$ \\
\hline Standard deviation & \pm 12.3 & \pm 10.4 & \pm 11.3 & \\
\hline Transplant, n (\%) & & & & $0.630^{a}$ \\
\hline Primary & $29(97)$ & $32(94)$ & $61(95)$ & \\
\hline Secondary & $1(3)$ & $2(6)$ & $3(5)$ & \\
\hline Recipient CMV status, n (\%) & & & & $0.244^{a}$ \\
\hline Positive & $27(90)$ & $33(97)$ & $60(94)$ & \\
\hline Primary etiology of renal failure, n (\%) & & & & $0.123^{a}$ \\
\hline Autoimmune disease & $1(3)$ & 0 & $1(2)$ & \\
\hline Diabetes mellitus & $1(3)$ & $4(12)$ & $5(8)$ & \\
\hline Failure of previous graft & 0 & $1(3)$ & $1(2)$ & \\
\hline Glomerulonephritis & $8(27)$ & $4(12)$ & $12(19)$ & \\
\hline Hypertension & $5(17)$ & 0 & $5(8)$ & \\
\hline IgA nephropathy (Berger's) & $2(7)$ & $3(9)$ & $5(8)$ & \\
\hline Interstitial nephritis/pyelonephritis & $4(13)$ & $6(18)$ & $10(16)$ & \\
\hline Other/unknown & $8(27)$ & $15(44)$ & $23(36)$ & \\
\hline Polycystic disease-kidney & $1(3)$ & $1(3)$ & $2(3)$ & \\
\hline Delayed graft function, $\mathrm{n}(\%)$ & & & & $0.442^{a}$ \\
\hline Yes & $4(13)$ & $7(21)$ & $11(17)$ & \\
\hline Acute rejection, $\mathrm{n}(\%)$ & & & & $0.209^{a}$ \\
\hline Yes & $1(3)$ & $4(12)$ & $5(8)$ & \\
\hline \multicolumn{5}{|l|}{ Number of HLA mismatches } \\
\hline Mean (range) & $3.4(1-5)$ & $3.4(1-5)$ & $3.4(1-5)$ & $0.991^{b}$ \\
\hline Standard deviation & \pm 1.2 & \pm 1 & \pm 1.1 & \\
\hline \multicolumn{5}{|l|}{ Ischemia time, hours } \\
\hline Mean (range) & $20.3(16-26)$ & $18.8(3-27)$ & $19.5(3-27)$ & $0.218^{b}$ \\
\hline Standard deviation & \pm 2.8 & \pm 5.7 & \pm 4.6 & \\
\hline \multicolumn{5}{|l|}{ Donor age, years } \\
\hline Mean (range) & $39.6(16-73)$ & $38.2(8-67)$ & $38.4(8-73)$ & $0.906^{b}$ \\
\hline Standard deviation & \pm 16.3 & \pm 16.5 & \pm 16.3 & \\
\hline Donor CMV status, n (\%) & & & & $0.639^{a}$ \\
\hline Positive & $25(86)$ & $27(82)$ & $52(84)$ & \\
\hline \multicolumn{5}{|l|}{ Quality of biopsy specimens (\%) } \\
\hline Adequate & 38.1 & 43.9 & 41 & $0.286^{b}$ \\
\hline Marginal & 29.7 & 26.3 & 28 & $0.320^{b}$ \\
\hline Unsatisfactory & 32.2 & 29.8 & 31 & $0.251^{b}$ \\
\hline
\end{tabular}

${ }^{a}$ Pearson chi-square test.

${ }^{b}$ Mann-Whitney test.

CMV, cytomegalovirus; Ig, immunoglobulin; HLA, human leukocyte antigen.

\section{Evaluation of Histologic Graft Damage}

Progression of any basic lesion was considered to be present when a Banff or CADI score was higher in the 1-year biopsy than in the pretransplant (baseline) biopsy of that patient, regardless of the severity of the lesion in the donor. When the score was equal to or lower than the pretransplant score, the patient was considered to have no progression of the lesions. Chronicity scores ranged from 0 to 12 for
Banff-CS and from 0 to 18 for the CADI score. These results were analyzed similarly for progression or no progression of scores for each patient (pair of biopsies) analyzed. Because Banff and CADI scores are semiquantitative parameters, the percentage of patients with progression of chronicity findings in each group (individual lesions and CS) was deemed the most reliable parameter by which to evaluate the severity of chronic graft damage. 


\section{Incidence and Severity of CAN}

We calculated the prevalence of lesions compatible with the diagnosis of CAN (according to Banff criteria) at the time of transplantation and at 1 year after transplantation, as well as the incidence of new cases of CAN that developed during the first year. According to the Banff classification, the diagnosis of CAN can be made when mild chronic interstitial (cil) and mild chronic tubular (ct1) changes are present. We also analyzed the severity of CAN using grade I, grade II, and grade III Banff criteria.

\section{Statistical Analysis}

Quantitative variables were described using the mean, the standard deviation, and the range for each treatment group. A Mann-Whitney test was used for testing statistical mean differences between groups. A nonparametric test was used because of the low sample size and to avoid the parametric assumption of normal distribution.

Qualitative variables were summarized with the category frequencies and percentage for each treatment group. A chi-square test was used for testing the statistical relation between the qualitative variables and the treatment groups. If the expected frequency of more than the $20 \%$ of total cells of the contingence table was less than 5, a Fisher exact test was used.

For multiple comparisons, a Bonferroni adjustment was made. The significance level for all statistical tests was 0.05 .

\section{RESULTS}

\section{Renal Function}

At 1 year after transplantation, or 9 months after randomization, plasma creatinine was significantly lower in group B than in group A (130.8 vs. $166.2 \mu \mathrm{mol} / \mathrm{L}$, respectively, $P<0.05)$.

\section{Quality of Biopsy Material}

The mean number of glomeruli in the graft biopsies was 15.2 \pm 17.9 (range 0-94), and the mean number of arteries was 2.1 \pm 1.6 (range $0-8$ ). According to Banff classification, $52(41 \%)$ biopsies fulfilled the criteria for adequate specimen with at least 10 glomeruli and 2 arteries; 36 (28\%) biopsies fulfilled the criteria for marginal specimen with more than 6 glomeruli and at least 1 artery; and $40(31 \%)$ biopsies were classified as unsatisfactory because of the presence of less than 7 glomeruli or no arteries present for histologic evaluation. The percentage of adequate, marginal, and unsatisfactory specimens was comparable in both groups (Table 1). Twentythree of the basal biopsies (of 64) were wedge biopsies with a similar distribution in both groups (12 in group A and 11 in group B).

\section{Progression of Pathologic Lesions: Banff Classification}

A higher proportion of patients showed progression (increase in severity) of chronic interstitial (ci), tubular $(c t)$, and vascular $(\mathrm{cv})$ lesions in group A compared with group B ( $70 \%$ vs. $40.9 \%$, respectively, for $c i, P<0.05 ; 70 \%$ vs. $47.8 \%$, respectively, for $c t, P<0.05$; and $29.4 \%$ vs. $25 \%$, respectively, for $c v, P=\mathrm{NS})$. Progression of chronic glomerular ( $c g$ ) lesions and arterial hyalinosis $(a h)$ was not significantly different between both treatment arms ( $9.1 \%$ vs. $0 \%$, respectively, for $c g$, $P=\mathrm{NS}$; and $19 \%$ vs. $10.5 \%$, respectively, for $a h, P=\mathrm{NS}$ ) (Fig. $1)$.

The differences in progression of chronic lesions became more evident when they were compared with the Banff chronicity score, the sum of chronic glomerular, interstitial, tubular, and vascular lesions. Figure 1 also shows the percentage of patients with progression of Banff-CS in both groups: $76.5 \%$ of patients in group A versus $31.3 \%$ in group B demonstrated progression of chronic lesions $(P=0.01)$.

No significant differences in Banff acute lesions were observed between treatment arms. Progression of interstitial infiltrate $(i)$ and glomerular changes $(g)$ were not more frequent in any group $(36.8 \%$ vs. $28.6 \%, P=\mathrm{NS}$, and $15 \%$ vs. $27.3 \%, P=$ NS, respectively). Minimal differences in tubular $(t)$ and vascular $(v)$ lesions were observed $(P=\mathrm{NS})$. No cases at 1-year fulfilled the criteria for the diagnosis of acute rejection, whereas in four cases, pathologic findings were consistent with the diagnosis of borderline changes ( 2 in group A and 2 in group B).

When unsatisfactory specimens were excluded from the analysis and only adequate and marginal cases were considered, the results were consistent with what was previously described. In particular, Banff chronic interstitial and tubular differences (progression of $c i$ and $c t$ ) were more pronounced (Table 2).

\section{Progression of Pathologic Lesions: CADI Classification}

Evaluation of biopsies using the CADI classification showed results similar to those analyzed using the Banff classification. Interstitial fibrosis $(F)$ and tubular atrophy $(T)$, progressed more frequently in group A patients $(68.4 \%$ vs. $40.9 \%$ for $F, P<0.05 ; 68.4 \%$ vs. $45.5 \%$ for $T, P<0.05)$. There

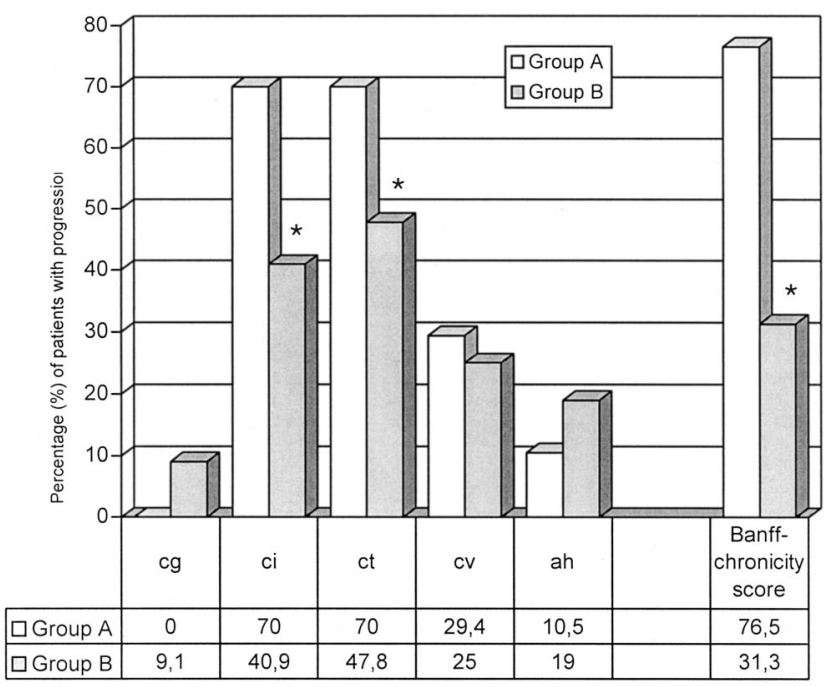

FIGURE 1. Percentage of patients with progression of Banff chronic individual lesions and Banff-chronicity score $(\mathrm{cg}+\mathrm{ci}+\mathrm{ct}+\mathrm{cv})$ from the time of transplantation to the first year after transplantation. $* P<0.05$ between groups. $\mathrm{cg}$, chronic glomerular damage; ci, interstitial fibrosis; ct, tubular fibrosis; cv, vascular intimal thickening. 
TABLE 2. Percentage of pathologic lesions in the subgroup of patients with only adequate or marginal biopsy specimens

\begin{tabular}{lccc} 
& Group A & Group B & P Value \\
\hline Banff-cg & 0 & 7.7 & NS \\
Banff-ci & 70 & 15 & $<0.05$ \\
Banff-ct & 70 & 23 & $<0.05$ \\
Banff-cr & 20 & 23 & NS \\
Banff-ah & 10 & 15 & NS \\
Banff-CS & 70 & 23 & $<0.05$ \\
CADI-I & 60 & 61 & NS \\
CADI-F & 70 & 15 & $<0.05$ \\
CADI-M & 40 & 31 & NS \\
CADI-G & 0 & 15 & NS \\
CADI-T & 70 & 23 & $<0.05$ \\
CADI-V & 20 & 23 & NS \\
CADI score & 70 & 38 & NS \\
\hline
\end{tabular}

NS, not significant; cg, chronic glomerular damage; ci, interstitial fibrosis; ct, tubular fibrosis; cv, vascular intimal thickening; CADI, Chronic Allograft Damage Index; I, inflammation; F, interstitial fibrosis; M, masangia matrix increase; $\mathrm{G}$, glomerular sclerosis; $\mathrm{T}$, tubular atrophy; $\mathrm{V}$, intimal proliferation.

were no significant differences in intimal proliferation $(V)$ $(31.3 \%$ vs. $23 \%, P=\mathrm{NS})$, glomerular changes $(G)(5.3 \%$ vs. $14.3 \%, P=\mathrm{NS})$, interstitial infiltration $(I)$, or mesangial changes $(M)$. CADI score results were similar to those of Banff-CS: $75 \%$ of patients in group A showed progression of CADI score versus $43.8 \%$ in group B $(P<0.05)$ (Fig. 2$)$.

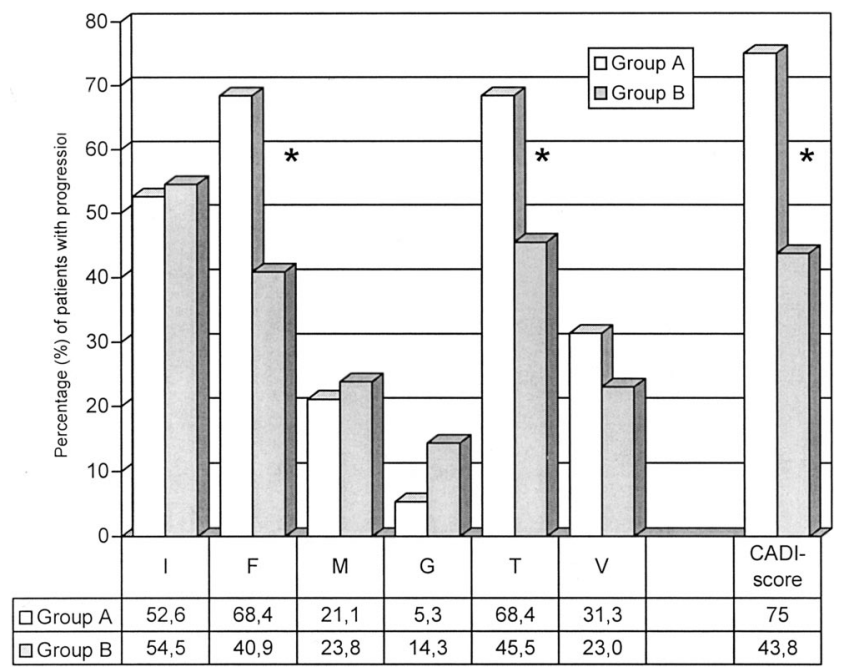

FIGURE 2. Percentage of patients with progression of CADI individual lesions and Chronic Allograft Damage In$\operatorname{dex}(\mathrm{CADI})$ score $(\mathrm{I}+\mathrm{F}+\mathrm{M}+\mathrm{G}+\mathrm{T}+\mathrm{V})$ from the time of transplantation to the first year after transplantation. $* P<0.05$ between groups. I, inflammation; F, interstitial fibrosis; $M$, mesangial matrix increase; G, glomerular sclerosis; T, tubular atrophy; $\mathrm{V}$, intimal proliferation.

\section{Incidence and Severity of CAN}

Using the Banff classification, the diagnosis of CAN (any grade) at 1 year after transplantation was higher, but not statistically significant, in the group remaining on CsA (70.8\% in group A and $59.3 \%$ in group $\mathrm{B} ; P=0.25$ ). The diagnosis of CAN was made more often in pretransplant biopsies in group B compared with group A $(21.7 \%$ vs. $9.5 \%$, respectively; $P=0.15$ ). However, $65 \%$ of patients in group A who did not have findings compatible with CAN in pretransplant biopsies developed changes consistent with CAN at 1 year, whereas only $31.8 \%$ of such cases in group B progressed to CAN at 1 year $(P<0.05)$. Furthermore, the severity of CAN at 1 year was higher in group A compared with group B ( $35.3 \%$ vs. $25 \%$, respectively, Banff grade III), although the difference was not statistically significant (Fig. 3).

\section{DISCUSSION}

The results of this study demonstrate that the elimination of CsA at 3 months after transplantation has a significant beneficial effect on the development and progression of chronic allograft damage during the first year after transplantation, despite the slightly higher rate of acute rejection observed in this group (10). This benefit may be a consequence of the prevention of chronic CsA toxicity.

The direct contribution of CNI toxicity to the progressive deterioration of renal function is difficult to establish. However, it has been demonstrated that renal function continues to improve for months after CNI discontinuation (13), which suggests a direct correlation that may be associated with a reduction in the production of profibrogenic mediators such as tumor growth factor (TGF)- $\beta$ (16). This mediator has been implicated in the development of tubulointerstitial fibrosis, the most characteristic finding associated with chronic CsA toxicity $(17,18)$.

The clinical results obtained from the RMR Study have clearly demonstrated that CsA elimination after the third postransplantation month is a safe procedure in the presence

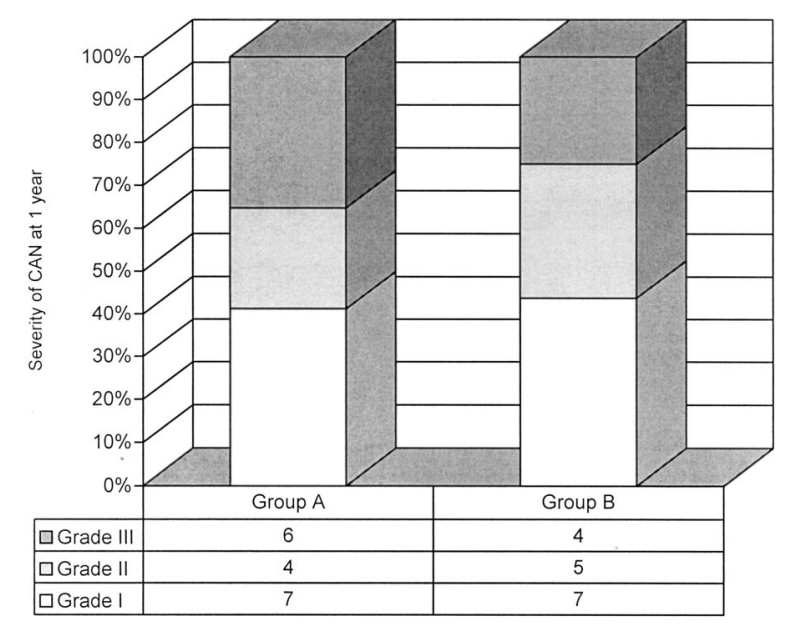

*No significant differences between groups.

FIGURE 3. Distribution of patients diagnosed with chronic allograft nephropathy (CAN) at 1 year after transplantation according to its severity. *No significant differences between groups. 
of SRL and results in an improvement in renal function (10). The 2-year results demonstrated continuous long-term improvement in renal function after CsA cessation, with the difference in renal function (mean serum creatinine) between the two treatment arms increasing from $15.7 \mu \mathrm{mol} / \mathrm{L}$ at 1 year to $28.4 \mu \mathrm{mol} / \mathrm{L}$ at 2 years (13). The hemodynamic effect of CsA elimination alone may not be responsible for this maintained and progressive improvement in renal function but may indicate a reduction in chronic graft CsA toxicity, which is manifested by chronic tubular and interstitial lesions. Furthermore, results from the Collaborative Transplant Study by Opelz et al. (19) indicate that improvement in blood pressure, as observed in the CsA elimination group (10), may be an independent factor responsible for better long-term kidneygraft outcome. Nevertheless, an improvement in long-term graft outcome should be associated with a reduction in graft pathologic lesions and a subsequent decrease in the incidence and severity of CAN. Our previous report suggested this hypothesis but the results were inconclusive because of limitations in the study design (11).

Our current study analyzed a subpopulation of the patients enrolled in the RMR Study, specifically those in Spain and Portugal. Approximately $80 \%$ of these patients had two biopsies performed. We performed a blinded reanalysis of most of these cases (64/70) that were also evaluated in the previous report, which corresponds to 128 of 140 biopsies. The pathologist received all samples at the same time and was unaware of treatment arm assignment as well as the time of biopsy. The blinded nature of the analysis is important because pathologists have a tendency to underestimate lesions they consider a consequence of pretransplant donor damage, which subsequently may influence the final results when comparing pretransplant and 1-year biopsies. All biopsies were reread in a short period of time, approximately 3 weeks. Surprisingly, the results obtained in this reanalysis, although similar to those published in the previous article, demonstrate more statistical significance. The criteria of interpretation from 10 different pathologists in the previous study could account for the difference in results.

One important factor to consider in the evaluation of chronic pathologic damage and the effect of immunosuppressive therapy is the presence of preexisting lesions in the donor that are indistinguishable from new lesions that develop after transplantation. The severity of such lesions can be correlated with the age of the donor and the existence of pathologic conditions such as arterial hypertension or diabetes mellitus. When comparing the severity of chronic changes in protocol biopsies at 1 year after transplantation between the two treatment arms, those pretransplant lesions must not be considered. For that reason, every case must be evaluated separately, comparing the severity of lesions in the 1-year biopsy with that in the baseline biopsy. Because lesions are evaluated using semiquantitative (not quantitative) scales (Banff and CADI), it is not possible to perform mathematical operations to subtract the value in the pretransplant biopsy from that in the 1-year biopsy. For that reason, we considered the existence of progression between biopsies in every lesion and then calculated the percentage of patients with progression as the optimal approach to the problem.

A potential shortcoming of our study is that we analyzed a smaller number of patients who represented a sub- group of patients from the RMR Study. However, we had a higher rate of patients who had two biopsies performed (80\%) compared with the other participating centers $(50 \%$ in the whole RMR Study). Also, the renal-function parameters of patients evaluated in this substudy were similar to those observed in our previous report (11). The direct effect of SRL in the reduction of chronic damage, because of its recognized antiproliferative effect, is difficult to asses in this study because both treatment arms received the drug (20). Nevertheless, it is important to consider that SRL levels after randomization in the third month were maintained at a higher level in group B, approximately two times that in group A, and this could be of some importance with respect to this effect.

Another potential shortcoming of the study is the risk of bias based on the actual distribution of adequate, marginal, and unsatisfactory biopsies between the two groups of patients. A significant imbalance in the proportion of unsatisfactory biopsies might be responsible for incorrect results. As Table 1 shows, this problem is clearly absent in our series because the distribution of the biopsy material in both groups according to quality is clearly comparable.

Stallone et al. (21) recently reported results similar to those presented in this study but in a smaller group of patients and with a less extensive biopsy analysis. Our study confirms the differences in the prevalence and severity of CAN but also demonstrated that the development of new cases after transplantation is increased when CsA is maintained, regardless of the severity of pathologic lesions in baseline biopsies. In addition, the analysis of individual pathologic lesions showed a significant benefit with CsA elimination in the rate of progression of tubular and interstitial lesions. These two lesions are associated with chronic CsA toxicity and are more directly related to the progressive deterioration of graft function. Nevertheless, we have no explanation for the absence of improvement in group B with respect to arteriolar hyalinosis, a lesion that clearly correlates with long-term CsA toxicity.

Analysis of acute lesions showed no differences between groups. Therefore, subclinical rejection with a subsequent risk of graft damage $(22,23)$ caused by suboptimal immunosuppression in patients who had CsA withdrawn was considered not to be present-this is a very interesting aspect-and contrasts with the results observed in some other trials where a reduced but significant number of cases show lesions compatible with subclinical rejection in protocol biopsies.

In conclusion, early elimination of CsA after transplantation in the presence of SRL results in a reduction both in the progression of pathologic lesions and in the appearance of new cases and severity of CAN, probably because of prevention of chronic nephrotoxicity. This benefit, combined with the improvement in renal function, may result in a subsequent improvement in long-term graft survival. Future results from the ongoing RMR Study (pathologic data at 3 years after transplant and clinical results at 5 years) will contribute significantly to the clarification of the benefits with calcineurin-free immunosuppression.

\section{ACKNOWLEDGMENTS}

The authors thank Ana Sanchez of the San Carlos Hospital, Madrid, Spain, for her help in statistical analysis and Susan 
Nastasee of Wyeth Research, Collegeville, PA, for her careful editorial review of this manuscript.

\section{REFERENCES}

1. Rigg KM. Renal transplantation: current status, complications and prevention. J Antimicrob Chemother 1995; 36(Suppl B): 51.

2. Solez K, Vincenti F, Filo RS. Histopathologic findings from 2-year protocol biopsies from a U.S. multicenter kidney transplant trial comparing tacrolimus versus cyclosporine: a report of the FK506 Kidney Transplant Study Group. Transplantation 1998; 66: 1736.

3. Paul LC. Chronic allograft nephropathy: an update. Kidney Int 1999; 56: 783.

4. Groth CG, Backman L, Morales JM, et al. Sirolimus (rapamycin)-based therapy in human renal transplantation: similar efficacy and different toxicity compared with cyclosporine. Sirolimus European Renal Transplant Study Group. Transplantation 1999; 67: 1036.

5. Kreis H, Cisterne JM, Land W, et al. Sirolimus in association with mycophenolate mofetil induction for the prevention of acute graft rejection in renal allograft recipients. Transplantation 2000; 69: 1252.

6. Flechner SM, Goldfarb D, Modlin C, et al. Kidney transplantation without calcineurin inhibitor drugs: a prospective, randomized trial of sirolimus versus cyclosporine. Transplantation 2002; 74: 1070.

7. Andoh TF, Lindsley J, Franceschini N, et al. Synergistic effects of cyclosporine and rapamycin in a chronic nephrotoxicity model. Transplantation 1996; 62: 311

8. Kahan BD. Efficacy of sirolimus compared with azathioprine for reduction of acute renal allograft rejection: a randomised multicentre study. The Rapamune US Study Group. Lancet 2000; 356: 194.

9. MacDonald AS. A worldwide, phase III, randomized, controlled, safety and efficacy study of a sirolimus/cyclosporine regimen for prevention of acute rejection in recipients of primary mismatched renal allografts. Transplantation 2001; 71: 271.

10. Johnson RW, Kreis H, Oberbauer R, et al. Sirolimus allows early cyclosporine withdrawal in renal transplantation resulting in improved renal function and lower blood pressure. Transplantation 2001; 72: 777.

11. Ruiz JC, Campistol JM, Mota A et al. Early cyclosporine a withdrawal in kidney transplant recipients under a sirolimus-based immunosuppres- sive regimen: pathological study of graft biopsies at 1-year posttransplant. Transplant Proc 2002; 34: 92.

12. Furness PN, Taub N. International variation in the interpretation of renal transplant biopsies: report of the CERTPAP Project. Kidney Int 2001; 60: 1998 .

13. Oberbauer R, Kreis H, Johnson RW, et al. Long-term improvement in renal function with sirolimus after early cyclosporine withdrawal in renal transplant recipients: 2-year results of the Rapamune Maintenance Regimen Study. Transplantation 2003; 76: 364.

14. Racusen LC, Solez K, Colvin RB, et al. The Banff 97 working classification of renal allograft pathology. Kidney Int 1999; 55: 713.

15. Isoniemi H, Taskinen E, Hayry P. Histological Chronic Allograft Damage Index accurately predicts chronic renal allograft rejection. Transplantation 1994; 58: 1195.

16. Shin GT, Khanna A, Ding R, et al. In vivo expression of transforming growth factor-betal in humans: stimulation by cyclosporine. Transplantation 1998; 65: 313 .

17. Andoh TF, Bennett WM. Chronic cyclosporine nephrotoxicity. Curr Opin Nephrol Hypertens 1998; 7: 265.

18. Bennett WM, Burdmann EA, Andoh TF, et al. Nephrotoxicity of immunosuppressive drugs. Nephrol Dial Transplant 1994; 9(Suppl 4): 141.

19. Opelz G, Wujciak T, Ritz E. Association of chronic kidney graft failure with recipient blood pressure. Collaborative Transplant Study. Kidney Int 1998; 53: 217.

20. Abraham RT. Mammalian target of rapamycin: immunosuppressive drugs uncover a novel pathway of cytokine receptor signaling. Curr Opin Immunol 1998; 10: 330.

21. Stallone G, Di Paolo S, Schena A, et al. Early withdrawal of cyclosporine A improves 1-year kidney graft structure and function in sirolimustreated patients. Transplantation 2003; 75: 998.

22. Shishido S, Asanuma H, Nakai H, et al. The impact of repeated subclinical acute rejection on the progression of chronic allograft nephropathy. J Am Soc Nephrol 2003; 14: 1046.

23. Kanetsuna $\mathrm{Y}$, Yamaguchi $\mathrm{Y}$, Toma $\mathrm{H}$, et al. Histological evaluation of renal allograft protocol biopsies in the early period and 1 year after transplantation. Clin Transplant 2003; 17(Suppl 10): 25. 